\title{
Role of Shear Wave Elastography in Evaluating the Risk of Hepatocellular Carcinoma in Patients with Chronic Hepatitis B
}

\author{
Jae Yoon Jeong ${ }^{1}$, Joo Hyun Sohn ${ }^{1}$, Won Sohn ${ }^{2}$, Chan Hyuk Park ${ }^{1}$, Tae Yeob Kim ${ }^{1}$, Dae Won Jun ${ }^{3}$, Yongsoo Kim ${ }^{4}$, and \\ Woo Kyoung Jeong ${ }^{5}$ \\ ${ }^{1}$ Department of Internal Medicine, Hanyang University Guri Hospital, Hanyang University College of Medicine, Guri, ${ }^{2}$ Hepatology Center, \\ Bundang Jesaeng General Hospital, Seongnam, ${ }^{3}$ Department of Internal Medicine, Hanyang University Hospital, Hanyang University College of \\ Medicine, Seoul, ${ }^{4}$ Department of Radiology, Hanyang University Guri Hospital, Hanyang University College of Medicine, Guri, and ${ }^{5}$ Department \\ of Radiology and Center for Imaging Science, Samsung Medical Center, Sungkyunkwan University School of Medicine, Seoul, Korea
}

Background/Aims: To investigate the use of measurements of liver stiffness (LS) by two-dimensional real-time shear wave elastography (SWE) for predicting the development of hepatocellular carcinoma (HCC) in patients with chronic hepatitis B (CHB). Methods: We retrospectively collected data on 291 enrolled patients with CHB whose LS had been measured using SWE. Results: The mean age of the patients was 46.8 years; males predominated (67\%), and 40 of the patients (14\%) had clinical cirrhosis. Among the patients, 165 (56.7\%) received antiviral treatment. The median LS value was $7.4 \mathrm{kPa}$, and the median followup period was 35.8 months (range, 3.0 to 52.8 months). During follow-up, HCC developed in 13 patients (4.5\%), and the cumulative incidence rates of $\mathrm{HCC}$ at 1, 2, and 4 years were $1.1 \%, 3.6 \%$, and $8.4 \%$, respectively. Based on a multivariate analysis, older age ( $\geq 50$ years) and higher LS value $(\geq 10 \mathrm{kPa}$ ) were independently associated with the risk of developing HCC (hazard ratio [HR], 4.53, $p=0.023$; and HR, 4.08, $p=0.022)$. The cumulative incidence rate of HCC was significantly higher in patients with higher LS values $(\geq 10$ $\mathrm{kPa})$ than in those with lower LS values $(<10 \mathrm{kPa})(\mathrm{p}=0.001)$. Conclusions: Increased LS measured by SWE at any time point regardless of antiviral treatment is associated with an increased risk of HCC in patients with CHB. (Gut Liver 2017;11:852-859)

Key Words: Elastography, shear wave; Liver stiffness; Hepatitis B, chronic; Carcinoma, hepatocellular

\section{INTRODUCTION}

Chronic hepatitis B virus (HBV) infection affected about 248 million individuals globally in 2010, and is the most common cause of end stage liver disease such as liver cirrhosis and hepatocellular carcinoma (HCC). ${ }^{1}$ Until now, the known risk factors for HCC development were older age, male gender, family history of HCC, alcohol consumption, high HBV DNA level and cirrhosis. ${ }^{2}$ Of these, cirrhosis was the most important risk factor. ${ }^{2}$ So, it is important to detect advanced fibrosis or cirrhosis early in patients with chronic hepatitis B (CHB), and these patients should be included in an optimized surveillance program for HCC.

To date, liver biopsy is considered the gold standard for evaluating liver fibrosis and cirrhosis. ${ }^{3}$ However, biopsy is a costly and invasive procedure that may be accompanied by complications, such as pain, hemorrhage and perforation. ${ }^{4}$ Hence the opportunity for repeated examinations is limited. Furthermore, liver biopsy is susceptible to sampling error and intra- and interobserver variability in interpretation. ${ }^{5}$ Because of these limitations, many noninvasive methods have been proposed as substitutes for liver biopsy. ${ }^{6}$

Over the last decade, elastography, especially transient elastography (TE) has been widely used to assess liver fibrosis and cirrhosis and portal hypertension-related complications in patients with chronic liver disease. ${ }^{7-9}$ In addition, TE has been shown to be a useful predictor of HCC development in CHB patients. ${ }^{10,11}$ Recently, Kim et al. ${ }^{12}$ reported that TE can identify CHB patients with so-called subclinical cirrhosis who are not clinically cirrhotic but have an increased risk of developing HCC.

\footnotetext{
Correspondence to: Joo Hyun Sohn

Department of Internal Medicine, Hanyang University Guri Hospital, 153 Gyeongchun-ro, Guri 11923, Korea

Tel: +82-31-560-2225, Fax: +82-31-555-2998, E-mail: sonjh@hanyang.ac.kr

Received on October 24, 2016. Revised on December 15, 2016. Accepted on January 17, 2017. Published online June 27, 2017

pISSN 1976-2283 eISSN 2005-1212 https://doi.org/10.5009/gnl16521

@) This is an Open Access article distributed under the terms of the Creative Commons Attribution Non-Commercial License (http://creativecommons.org/licenses/by-nc/4.0) which permits unrestricted non-commercial use, distribution, and reproduction in any medium, provided the original work is properly cited.
} 
Second-generation elastography procedures known as acoustic radiation force impulse imaging and two-dimentional real-time shear wave elastography (SWE) are subsequently introduced and actively used worldwide. ${ }^{13-15}$ Unlike TE, LS measurement by SWE is guided by a B-mode image and assesses precisely the degree of liver fibrosis. ${ }^{16}$ Several studies have reported that SWE is as good as or better than TE for assessing liver fibrosis. ${ }^{14,17,18}$ However, there have been few studies using LS measured by SWE to predict HCC development in patients with $\mathrm{CHB} .{ }^{2}$ We therefore performed this study to investigate the potential of SWE for predicting HCC in patients with HBVrelated chronic hepatitis or compensated cirrhosis.

\section{MATERIALS AND METHODS}

\section{Patients}

LS was measured by SWE in 446 patients with CHB from January 2011 to December 2012 at Hanyang University Guri Hospital, Guri, Korea. Of these, 155 patients were excluded for the following reasons (Fig. 1): (1) invalid LS value ( $n=2),(2)$ past history of decompensation ( $\mathrm{n}=24)$, (3) Child-Pugh class B or C ( $n=11),(4)$ HCC at enrollment, or past history of HCC ( $n=21),(5)$ co-infection with other hepatitis viruses $(n=1),(6)$ pregnancy $(n=2)$, and (7) loss to follow-up before HCC surveillance $(n=94)$. Finally, 291 patients with CHB were included in the analysis. According to the Korea association for the Study of the Liver guideline, hepatitis B e antigen ( $\mathrm{HBeAg}$ )-positive patients with an HBV DNA level of $\geq 20,000 \mathrm{IU} / \mathrm{mL}$ and an alanine transaminase (ALT) level of $\geq 2 \times$ upper normal limit (UNL), HBeAg-negative patients with an HBV DNA level of $\geq 2,000 \mathrm{IU} / \mathrm{mL}$ and an ALT level of $\geq 2 \times U N L$, and compensated cirrhotic patients with an HBV DNA level of $\geq 2,000 \mathrm{IU} / \mathrm{mL}$ regardless of the ALT level were administered to antiviral agents. ${ }^{19}$ Our study was approved by the Ethics Committee of Hanyang University Guri Hospital

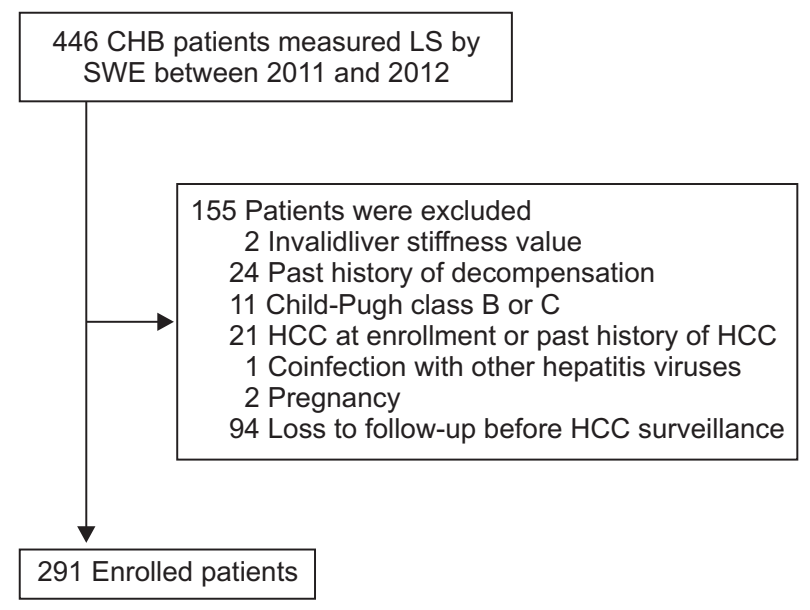

Fig. 1. Flow chart of enrolled patients. CHB, chronic hepatitis B; LS, liver stiffness; SWE, shear wave elastography; HCC, hepatocellular carcinoma.
(IRB number: 2015-11-011-001).

\section{Clinical and laboratory parameters}

We collected the following clinical and laboratory data; age, gender, clinical cirrhosis, previous or ongoing antiviral treatment, alcohol abuse, diabetes mellitus, family history of HCC, body mass index (BMI), platelet (PLT) count, albumin, total bilirubin, prothrombin time (international normalized ratio, INR), aspartate transaminase (AST), ALT, HBsAg, HBeAg, HBeAb, HBV DNA and LS measurement by SWE. Additionally FIB-4 index ([agexAST]/[PLT count $\left(\times 10^{9} / \mathrm{L}\right) \times \sqrt{ }$ ALT] $)$ and APRI (AST/ upper limit of normal/PLT count $\left.\left[\times 10^{9} / \mathrm{L}\right] \times 100\right)$ were calculated using the same blood. Clinical cirrhosis was defined as (1) a PLT count $<100,000 / \mathrm{mm}^{2}$ and ultrasonographic findings suggestive of cirrhosis, including surface nodularity with splenomegaly (>12 $\mathrm{cm}$ ) and (2) presence of esophageal or gastric varices. ${ }^{11}$ Alcohol abuse was defined as ingestion of $>30 \mathrm{~g} /$ day of alcohol in men and $>20 \mathrm{~g} /$ day in women. All patients underwent measurement of baseline serum laboratory parameters within 3 months of the first elastography imaging, and then every 3 to 6 months. Serum HBV DNA levels were measured by real-time PCR (Roche Diagnostics, Branchburg, NJ, USA; detection range: $20 \sim 1 \times 10^{9}$ $\mathrm{IU} / \mathrm{mL}$ ). In our study, 25 percentile, median, and 75 percentile of LS value were 5.9, 7.4, and $10.2 \mathrm{kPa}$, respectively. Thus, the patients with LS $\geq 10 \mathrm{kPa}$ (73.5 percentile) could be regarded as the high risk group. For this, we used $10 \mathrm{kPa}$ as the cutoff value of LS for HCC development risk.

\section{Patient follow-up}

Patients were screened for HCC every 6 to 12 months by $\alpha$-fetoprotein assay and ultrasonography, or dynamic computed tomography. A diagnosis of HCC was established according to the Guidelines of the American Association for the Study of Liver Diseases. ${ }^{20}$ The last follow-up date was June 2015.

\section{Liver stiffness measurement by SWE}

For LS measurement an Aixplorer ultrasound (US) system (Supersonic Imagine SA, Aix-en-Provence, France) equipped with an elastographic function was used. SWE is based on a two-dimensional elastography technique. All LS measurement were performed after conventional liver US by two abdominal radiologists (Y.K. and W.K.J.) with over 10 years of clinical experience in abdominal radiology and liver US as part of their regular practice. They also had experience of performing LS measurement in more than 100 patients. For the LS measurements, hepatic ultrasonograms were obtained via a right intercostal window, with 5- to 10-repetition per session, and the median value was taken as the LS measurement. Ultrasonograms were acquired at a location more than $2 \mathrm{~cm}$ below the hepatic capsule, and the region-of-interest was located away from large vessels to avoid measurement error. Unreliable LS measurement due to noncooperation or a thick subcutaneous layer of fat was 
considered as invalid LS measurement.

\section{Statistical analysis}

Categorical variables are given as frequencies and percentages. Continuous variables are given as mean values with standard deviations and median values with ranges for parametric and nonparametric variables, respectively. The cumulative incidence rate of HCC was calculated using the Kaplan-Meier method. Time-dependent areas under the receiver operating characteristics (AUROC) using LS measurement was assessed to predict HCC development. To assess risk factors for HCC development, univariate and multivariable Cox proportional hazards models were used. The variables for a multivariable analysis were determined on the basis of their statistical significance in the univariate analysis $(\mathrm{p}<0.10)$. A backward conditional stepwise procedure was performed in the multivariable analysis to avoid multicollinearity. Independent risk factors were compared using the log-rank test. The p-values $<0.05$ in two-sided tests were considered statistically significant. SPSS 18.0 for Windows (SPSS Inc., Chicago, IL, USA) was used for all statistical analysis except time-dependent AUROC. Time-dependent AUROC was calculated using R 3.1.1 (Vienna, Austria; http://www.R-project. org).

\section{RESULTS}

\section{Baseline characteristics}

The baseline characteristics of the 291 patients are summarized in Table 1 . Their mean age was 46.8 years and they were predominantly male $(n=195,67 \%)$. One hundred sixty-five patients (56.7\%) received previous or ongoing antiviral treatment. Clinical cirrhosis was identified in 40 (14\%). Thirty-two patients had alcohol abuse and 23 had diabetes mellitus. Mean BMI and ALT were $24.2 \mathrm{~kg} / \mathrm{m}^{2}$ and $53.8 \mathrm{U} / \mathrm{L}$, respectively. In addition, the mean values of APRI and FIB-4 index were 0.8 and 2.0, respectively. The median value of LS was $7.4 \mathrm{kPa}$ (range, 3.4 to 36.0 $\mathrm{kPa})$.

\section{HCC and liver-related events}

The median follow-up period was 35.8 months (range, 3.0 to 52.8 months) (784.9 person-years of follow-up). During follow up, 13 patients (4.5\%) developed HCC. According to the modified staging system of the International Union against Cancer, there were four (30.8\%), six (46.2\%), two (15.4\%), and one (7.7\%) stage I, II, III and IVa patients, respectively. During the followup period, four patients developed liver-related complications other than HCC and all were ascites. The LS values of the four patients who developed ascites were 9.83, 12.15, 12.25 and $13.12 \mathrm{kPa}$, respectively, and all had clinical cirrhosis. One of these patients developed HCC later.
Table 1. Baseline Characteristics of the Enrolled Patients $(n=291)$

\begin{tabular}{|c|c|}
\hline Variable & Value \\
\hline Age, yr & $46.8 \pm 11.4$ \\
\hline \multicolumn{2}{|l|}{ Sex } \\
\hline Male & $195(67)$ \\
\hline Female & $96(33)$ \\
\hline Clinical cirrhosis & $40(14)$ \\
\hline Antiviral treatment & $165(57)$ \\
\hline Alcohol abuse & $32(11)$ \\
\hline Diabetes mellitus & $23(8)$ \\
\hline Family history of HCC & $45(16)$ \\
\hline BMI, $\mathrm{kg} / \mathrm{m}^{2}$ & $24.2 \pm 3.1$ \\
\hline Platelet count, $\times 10^{3} / \mathrm{mm}^{2}$ & $172.7 \pm 53.1$ \\
\hline Albumin, g/dL & $4.4 \pm 0.4$ \\
\hline Total bilirubin, mg/dL & $0.9 \pm 1.0$ \\
\hline Prothrombin time, INR & $0.9 \pm 0.1$ \\
\hline AST, U/L & $46.3 \pm 124.4$ \\
\hline ALT, U/L & $53.8 \pm 119.8$ \\
\hline HBeAg positivity & $119(41)$ \\
\hline HBV DNA, IU/mL & 110.0 (undetectable-170,000,000) \\
\hline APRI & $0.8 \pm 2.3$ \\
\hline FIB-4 & $2.0 \pm 1.9$ \\
\hline LS by SWE, $\mathrm{kPa}$ & $7.4(3.4-36.0)$ \\
\hline
\end{tabular}

Data are presented as mean $\pm \mathrm{SD}$, number (\%), or median (range). HCC, hepatocellular carcinoma; BMI, body mass index; INR, international normalized ratio; AST, aspartate aminotransferase; ALT, alanine aminotransferase; HBeAg, hepatitis B e antigen; HBV, hepatitis $B$ virus; APRI, AST to platelet ratio index; FIB-4, fibrosis-4 index; LS, liver stiffness; SWE, shear wave elastography.

\section{Comparison between patients who did and did not develop HCC}

The baseline characteristics of the patients who did and did not develop HCC are shown in Table 2. The mean LS value by SWE was significantly higher in patients who did develop HCC than in those who did not (12.2 kPa vs $7.3 \mathrm{kPa}, \mathrm{p}=0.007)$. Age was also significantly higher in patients who did develop HCC than in those who did not (57.2 years vs 46.4 years, $\mathrm{p}<0.001$ ), but PLT count was lower $\left(123 \times 10^{3} / \mathrm{mm}^{2}\right.$ vs $175 \times 10^{3} / \mathrm{mm}^{2}$, $\mathrm{p}<0.001)$.

\section{Independent predictors of HCC development}

In univariate analysis, HCC development was significantly associated with older age ( $\geq 50$ years) (hazard ratio [HR], 5.39; $\mathrm{p}=0.010)$, clinical cirrhosis (HR, 4.76; $\mathrm{p}=0.005)$, lower PLT counts $\left(<150 \times 10^{3} / \mathrm{mm}^{2}\right)$ (HR, 3.15; $\left.\mathrm{p}=0.044\right)$, higher total bilirubin ( $\geq 1.0 \mathrm{mg} / \mathrm{dL}$ ) (HR, 3.34; $\mathrm{p}=0.031$ ), FIB-4 $\geq 3.25$ (HR, 7.77; $\mathrm{p}<0.001)$ and higher LS values $(\geq 10 \mathrm{kPa})(\mathrm{HR}, 5.97 ; \mathrm{p}=0.003)$ (Table 3). In multivariable analysis, older age ( $\geq 50$ years) and higher LS value ( $\geq 10 \mathrm{kPa}$ ) were independently associated with 
Table 2. Comparison of the Baseline Characteristics of Chronic Hepatitis B Patients with and without Hepatocellular Carcinoma Development

\begin{tabular}{|c|c|c|c|}
\hline Variable & $\begin{array}{c}\text { Non-HCC } \\
(\mathrm{n}=278,95.5 \%)\end{array}$ & $\begin{array}{c}\text { HCC } \\
(n=13,4.5 \%)\end{array}$ & $\mathrm{p}$-value \\
\hline Age, yr & $46.4 \pm 11.3$ & $57.2 \pm 8.1$ & 0.001 \\
\hline Sex & & & 0.862 \\
\hline Male & $186(67)$ & $9(69)$ & \\
\hline Female & $92(33)$ & $4(31)$ & \\
\hline Clinical cirrhosis & $34(12)$ & $6(46)$ & 0.001 \\
\hline Antiviral treatment & $157(56)$ & $8(61)$ & 0.719 \\
\hline Alcohol abuse & $30(11)$ & $2(15)$ & 0.609 \\
\hline Diabetes mellitus & $22(8)$ & $1(8)$ & 0.974 \\
\hline Family history of HCC & $42(15)$ & $3(23)$ & 0.445 \\
\hline BMI, $\mathrm{kg} / \mathrm{m}^{2}$ & $24.1 \pm 3.1$ & $25.7 \pm 3.4$ & 0.086 \\
\hline Platelet count, $\times 10^{3} / \mathrm{mm}^{2}$ & $175.0 \pm 52.3$ & $123.2 \pm 46.8$ & 0.001 \\
\hline Albumin, $\mathrm{g} / \mathrm{dL}$ & $4.4 \pm 0.4$ & $4.4 \pm 0.3$ & 0.556 \\
\hline Total bilirubin, mg/dL & $0.9 \pm 1.0$ & $0.9 \pm 0.2$ & 0.992 \\
\hline Prothrombin time, INR & $0.9 \pm 0.1$ & $1.0 \pm 0.1$ & 0.679 \\
\hline AST, U/L & $46.3 \pm 127.2$ & $39.1 \pm 17.0$ & 0.831 \\
\hline ALT, U/L & $54.8 \pm 122.4$ & $32.1 \pm 22.9$ & 0.505 \\
\hline HBeAg positivity & $114(41)$ & $5(39)$ & 0.855 \\
\hline HBV DNA, IU/mL & 110.0 (undetectable-170,000,000) & 32.6 (undetectable-37,100,000) & 0.559 \\
\hline APRI & $0.8 \pm 2.3$ & $1.1 \pm 1.1$ & 0.673 \\
\hline FIB-4 & $1.9 \pm 1.8$ & $4.1 \pm 2.9$ & 0.019 \\
\hline LS by SWE, $\mathrm{kPa}$ & $7.3(3.4-36.0)$ & $12.2(7.1-23.8)$ & 0.007 \\
\hline
\end{tabular}

Data are presented as mean $\pm \mathrm{SD}$, number $(\%)$, or median (range).

HCC, hepatocellular carcinoma; BMI, body mass index; INR, international normalized ratio; AST, aspartate aminotransferase; ALT, alanine aminotransferase; HBeAg, hepatitis B e antigen; HBV, hepatitis B virus; APRI, AST to platelet ratio index; FIB-4, fibrosis-4 index; LS, liver stiffness; SWE, shear wave elastography.

risk of developing HCC (HR, 4.53, p=0.023; and HR, 4.08, $\mathrm{p}=0.022$ ) (Table 3).

\section{Incidence of HCC development according to risk factors}

Cumulative rates of HCC at 1-, 2-, and 4-year were 1.1\%, 3.6\%, and $8.4 \%$, respectively, for all patients. It was significantly higher in patients with higher LS values $(\geq 10 \mathrm{kPa}$ ) than those with lower LS values $(<10 \mathrm{kPa})$ (log-rank test, $\mathrm{p}=0.001)$ (Fig. $2 \mathrm{~B})$. The cumulative rates of HCC at 1-, 2-, and 4-years were 0.5\%, 1.6\%, and $2.5 \%$, respectively, in patients with LS values $<10 \mathrm{kPa}$, and $2.6 \%, 5.5 \%$, and $21.5 \%$, respectively, in patients with LS scores $\geq 10 \mathrm{kPa}$. The time-dependent AUROCs of LS measurement by SWE for HCC development at 1-, 2-, 3-, 4-, and 5-year were $0.756,0.712,0.772,0.747$, and 0.747 , respectively.

HCC development differed significantly according to age (logrank test, $p=0.004$ ) (Fig. 2A). The cumulative rates of HCC at 1-, $2-$, and 4-year were $0 \%, 1.2 \%$, and $4.4 \%$, respectively, in patients with age $<50$ years, and 2.9\%, 5.0\%, and 14.5\%, respectively, in patients with age $\geq 50$ years.

\section{DISCUSSION}

We have investigated the potential of LS measured by SWE as a new shear wave-based elastography technique for predicting HCC development in patients with HBV-related chronic hepatitis or compensated cirrhosis. We have shown that the risk of HCC development increased up to 4-fold in CHB patients with LS $\geq 10 \mathrm{kPa}$ by SWE compared to those with LS $<10 \mathrm{kPa}$, and the incidence of HCC in CHB patients with LS $\geq 10 \mathrm{kPa}$ was significantly higher than in those with $\mathrm{LS}<10 \mathrm{kPa}$. These results show that increased LS values assessed by SWE at any time point regardless of antiviral treatment are associated with an increased risk of HCC, and suggest that SWE is useful as a new method for evaluating the risk of HCC in compensated patients with $\mathrm{CHB}$.

In recent years TE has been used as a popular noninvasive method to predict degrees of liver fibrosis and cirrhosis. ${ }^{7,21}$ Also many studies have reported that LS measurement by TE is a useful test for HCC development in patient with CHB. ${ }^{11,12,22}$ However, because of several limitations LS measurement by TE cannot be performed in all patients. Also LS measurement by TE 
Table 3. Factors Predicting Hepatocellular Carcinoma Development in Compensated Patients with Chronic Hepatitis B

\begin{tabular}{|c|c|c|c|c|}
\hline Variable & $\begin{array}{c}\text { Univariate HR } \\
(95 \% \mathrm{CI})\end{array}$ & p-value & $\begin{array}{c}\text { Multivariable HR } \\
(95 \% \mathrm{CI})\end{array}$ & p-value \\
\hline \multicolumn{5}{|l|}{ Sex } \\
\hline Female & 1 & & & \\
\hline Male & $1.11(0.34-3.61)$ & 0.864 & & \\
\hline \multicolumn{5}{|l|}{ Age, yr } \\
\hline$<50$ & 1 & & 1 & \\
\hline$\geq 50$ & $5.39(1.48-19.81)$ & 0.010 & 4.53 (1.23-16.67) & 0.023 \\
\hline \multicolumn{5}{|l|}{ Clinical cirrhosis } \\
\hline Absent & 1 & & & \\
\hline Present & $4.76(1.60-14.19)$ & 0.005 & & \\
\hline \multicolumn{5}{|l|}{ Antiviral treatment } \\
\hline No & 1 & & & \\
\hline Yes & $1.12(0.37-3.43)$ & 0.840 & & \\
\hline \multicolumn{5}{|l|}{ Alcohol abuse } \\
\hline Absent & 1 & & & \\
\hline Present & $1.45(0.32-6.56)$ & 0.626 & & \\
\hline \multicolumn{5}{|l|}{ Diabetes mellitus } \\
\hline Absent & 1 & & & \\
\hline Present & $1.11(0.14-8.54)$ & 0.921 & & \\
\hline \multicolumn{5}{|l|}{ Family history of HCC } \\
\hline Absent & 1 & & & \\
\hline Present & $1.58(0.44-5.75)$ & 0.486 & & \\
\hline \multicolumn{5}{|l|}{ BMI, kg/m² } \\
\hline$<25$ & 1 & & & \\
\hline$\geq 25$ & $2.46(0.81-7.52)$ & 0.114 & & \\
\hline \multicolumn{5}{|c|}{ Platelet count, $\times 10^{3} / \mathrm{mm}^{2}$} \\
\hline$\geq 150$ & 1 & & & \\
\hline$<150$ & $3.15(1.03-9.66)$ & 0.044 & & \\
\hline \multicolumn{5}{|l|}{ Albumin, mg/dL } \\
\hline$\geq 4.0$ & 1 & & & \\
\hline$<4.0$ & $1.96(0.43-8.84)$ & 0.383 & & \\
\hline \multicolumn{5}{|l|}{ Total bilirubin, mg/dL } \\
\hline$<1.0$ & 1 & & & \\
\hline$\geq 1.0$ & $3.34(1.11-9.99)$ & 0.031 & & \\
\hline \multicolumn{5}{|c|}{ Prothrombin time, INR } \\
\hline$<1.0$ & 1 & & & \\
\hline$\geq 1.0$ & $0.84(0.19-3.81)$ & 0.822 & & \\
\hline \multicolumn{5}{|l|}{ AST, U/L } \\
\hline$<40$ & 1 & & & \\
\hline$\geq 40$ & $2.37(0.77-7.26)$ & 0.131 & & \\
\hline \multicolumn{5}{|l|}{ ALT, U/L } \\
\hline$<40$ & 1 & & & \\
\hline$\geq 40$ & $0.53(0.12-2.37)$ & 0.402 & & \\
\hline \multicolumn{5}{|l|}{ HBeAg positivity } \\
\hline Absent & 1 & & & \\
\hline Present & $1.05(0.34-3.22)$ & 0.930 & & \\
\hline \multicolumn{5}{|l|}{ HBV DNA, IU/mL } \\
\hline$<2,000$ & 1 & & & \\
\hline$\geq 2,000$ & $0.93(0.29-3.04)$ & 0.907 & & \\
\hline \multicolumn{5}{|l|}{ APRI } \\
\hline$<1.0$ & 1 & & & \\
\hline$\geq 1.0$ & $2.70(0.93-9.81)$ & 0.099 & & \\
\hline \multicolumn{5}{|l|}{ FIB-4 index } \\
\hline$<3.25$ & 1 & & & \\
\hline$\geq 3.25$ & 7.77 (2.39-25.27) & 0.001 & & \\
\hline LS by SWE, kPa & & & & \\
\hline$<10$ & 1 & & 1 & \\
\hline$\geq 10$ & $5.97(1.84-19.41)$ & 0.003 & $4.08(1.23-13.55)$ & 0.022 \\
\hline
\end{tabular}

HR, hazard ratio; CI, confidence interval; HCC, hepatocellular carcinoma; BMI, body mass index; INR, international normalized ratio; AST, aspartate aminotransferase; ALT, alanine aminotransferase; HBeAg, hepatitis B e antigen; HBV, hepatitis B virus; APRI, AST to platelet ratio index; FIB4, fibrosis-4 index; LS, liver stiffness; SWE, shear wave elastography. 
A

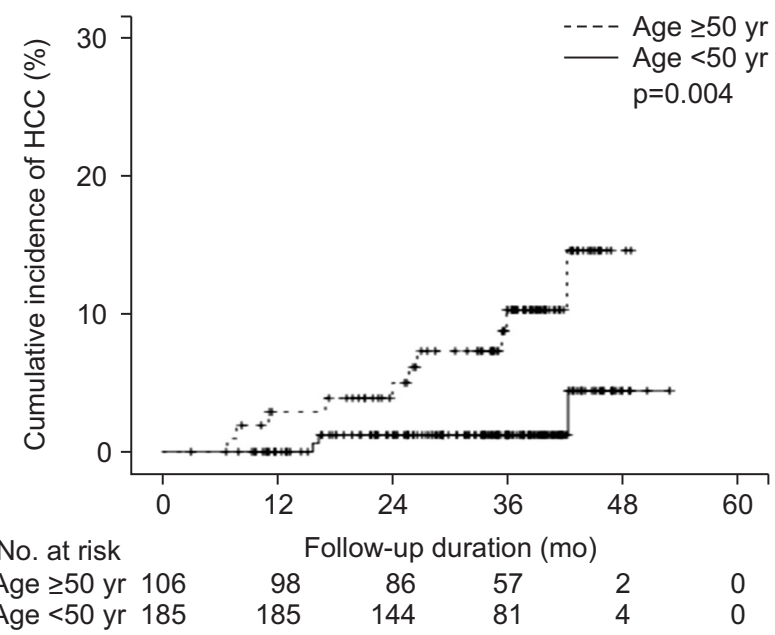

B



Fig. 2. The difference in hepatocellular carcinoma (HCC) incidence according to age (A) and liver stiffness based on shear wave elastography (SWE) (B).

can give unreliable results because of the bulky external vibrator. ${ }^{18}$ On the other hand, when LS measurement by SWE is used, the shear modulus of the shear wave generated by the acoustic radiation force impulses without requiring an external vibrator, and has good reliability and reproducibility. ${ }^{18}$ Also, SWE provides information on shear wave propagation over a larger area than TE. ${ }^{16}$ Furthermore, unlike SWE, TE cannot measure LS in patients with severe obesity, subcutaneous fat, or ascites because of poor penetration. ${ }^{23}$

Cirrhosis is the most important predictor of HCC development in CHB patients. ${ }^{2}$ Cirrhosis is commonly diagnosed from radiologic and laboratory findings, or from clinical findings such as varices, ascites, jaundice and hepatic encephalopathy. ${ }^{24}$ But if the findings are not typical it is difficult to diagnose cirrhosis. Also, since the findings are dichotomized, it is difficult to detect early cirrhosis or advanced fibrosis in patients with CHB. Hence an accurate surrogate for diagnosing cirrhosis is needed and it should be a quantitative measure. Jung et al. ${ }^{11}$ suggested that LS measurement by TE might be a more reliable method for diagnosing compensated cirrhosis than other clinical findings. As expected, our study showed that the risk of HCC development increased up to 4-fold in $\mathrm{CHB}$ patients with $\mathrm{LS} \geq 10 \mathrm{kPa}$ by SWE compared to those with LS $<10 \mathrm{kPa}$ when we applied $10 \mathrm{kPa}$ as the cutoff value. The reasons why we determined the 10 cutoff value of LS were as follows: First, as aforementioned 75 percentile of LS value was $10.2 \mathrm{kPa}$, so the patients with LS $\geq 10 \mathrm{kPa}$ (73.5 percentile) could be regarded as the high risk group. Second, when AUROC were used to calculate the optimal LS cutoff value for HCC development risk, the LS cutoff value was actually $9.4 \mathrm{kPa}$ which was closely with $10 \mathrm{kPa}$, and AUROC was 0.783 (95\% CI, 0.692 to 0.875 ; $p=0.001$; sensitivity, 76.9\%; specificity, $73.0 \%)$. Furthermore, the cutoff values of LS measured by SWE for liver cirrhosis by biopsy-proven histology in patients with
CHB were 10.1 and $11.7 \mathrm{kPa}$ in two recent studies. ${ }^{18,25}$ These implied that the patients with LS $\geq 10 \mathrm{kPa}$ in our study might have advanced fibrosis or cirrhosis. However, clinical cirrhosis was identified in only 40 among 78 patients (51.3\%) with a high LS value ( $\geq 10 \mathrm{kPa}$ ) in our study (Fig. $2 \mathrm{~B}$ ). These results indicated that SWE could help distinguish patients with a high risk of HCC earlier before clinical diagnosis of cirrhosis. Therefore we suggest that, like TE, SWE is a more reliable method than clinical criteria for predicting HCC development.

As high LS values, older age ( $>50$ years) was a risk factor for HCC development. This result is consistent with previous reports. ${ }^{12,22}$ However, a high HBV DNA load, which was considered an important risk factor for HCC development before the era of antiviral therapy, ${ }^{26}$ was not a risk factor in our study. For the last few years antiviral agents with high potency and low resistance such as entecavir and tenofovir have been extensively administered to CHB patients, and high viral loads were easily suppressed. ${ }^{27,28}$ Hence high viral load may no longer be a risk factor for HCC development in CHB patients who are regularly followed up. ${ }^{29}$

In our study four patients developed liver-related complications other than HCC and all were ascites. HCC was the leading liver-related complication of compensated liver cirrhosis with CHB receiving antiviral treatment in several studies. ${ }^{29,30}$ Recently Lampertico et al..$^{30}$ followed up $107 \mathrm{HBeAg-negative} \mathrm{compen-}$ sated cirrhotic patients during a median of 12 years of antiviral therapy and no patient experienced liver-related complications other than HCC. So we think that HCC may be the most common liver-related complication of compensated patients with CHB in the era of antiviral therapy of high potency and low resistance. Therefore it becomes more important to identify those CHB patients without clinically apparent cirrhosis who have an increased risk of HCC development. Our study indicates that, 
like TE, SWE is useful as a method of evaluating the risk of HCC development in those patients.

This study had several limitations. First, it was a retrospective study involving a relatively small number of patients in a single center. As a result, we could not demonstrate significant correlations between HCC development and other risk factors besides older age and high LS value. Second, since liver biopsy data were not available upon enrollment in most patients, we could not examine any correlations between LS values and detailed histologic findings such as fibrosis, inflammation and steatosis. We believe that a large and long-term prospective study is needed to confirm a robust association between HCC development and LS measured by SWE in patients with CHB.

In conclusion, we found that high LS values measured by SWE at any time point regardless of antiviral treatment are associated with an increased risk of HCC development in compensated patients with CHB. We therefore suggest that SWE could have an important role in evaluating the risk of HCC development in patients with $\mathrm{CHB}$.

\section{CONFLICTS OF INTEREST}

No potential conflict of interest relevant to this article was reported.

\section{REFERENCES}

1. Schweitzer A, Horn J, Mikolajczyk RT, Krause G, Ott JJ. Estimations of worldwide prevalence of chronic hepatitis B virus infection: a systematic review of data published between 1965 and 2013. Lancet 2015;386:1546-1555.

2. Wong VW, Janssen HL. Can we use HCC risk scores to individualize surveillance in chronic hepatitis B infection? J Hepatol 2015;63:722-732.

3. Dienstag JL. The role of liver biopsy in chronic hepatitis C. Hepatology 2002;36(5 Suppl 1):S152-S160.

4. Bravo AA, Sheth SG, Chopra S. Liver biopsy. N Engl J Med 2001; 344:495-500.

5. Bedossa P, Dargère D, Paradis V. Sampling variability of liver fibrosis in chronic hepatitis C. Hepatology 2003;38:1449-1457.

6. Yoshioka K, Hashimoto S. Can non-invasive assessment of liver fibrosis replace liver biopsy? Hepatol Res 2012;42:233-240.

7. Friedrich-Rust M, Ong MF, Martens S, et al. Performance of transient elastography for the staging of liver fibrosis: a meta-analysis. Gastroenterology 2008;134:960-974.

8. Robic MA, Procopet B, Métivier S, et al. Liver stiffness accurately predicts portal hypertension related complications in patients with chronic liver disease: a prospective study. J Hepatol 2011;55:10171024.

9. Pang JX, Zimmer S, Niu S, et al. Liver stiffness by transient elastography predicts liver-related complications and mortality in patients with chronic liver disease. PLoS One 2014;9:e95776.
10. Fung J, Lai CL, Seto WK, Wong DK, Yuen MF. Prognostic significance of liver stiffness for hepatocellular carcinoma and mortality in HBeAg-negative chronic hepatitis B. J Viral Hepat 2011;18:738744.

11. Jung KS, Kim SU, Ahn SH, et al. Risk assessment of hepatitis B virus-related hepatocellular carcinoma development using liver stiffness measurement (FibroScan). Hepatology 2011;53:885-894.

12. Kim MN, Kim SU, Kim BK, et al. Increased risk of hepatocellular carcinoma in chronic hepatitis B patients with transient elastography-defined subclinical cirrhosis. Hepatology 2015;61:1851-1859.

13. Friedrich-Rust M, Nierhoff J, Lupsor M, et al. Performance of acoustic radiation force impulse imaging for the staging of liver fibrosis: a pooled meta-analysis. J Viral Hepat 2012;19:e212-e219.

14. Ferraioli G, Tinelli C, Dal Bello B, et al. Accuracy of real-time shear wave elastography for assessing liver fibrosis in chronic hepatitis $\mathrm{C}$ : a pilot study. Hepatology 2012;56:2125-2133.

15. Singh S, Venkatesh SK, Wang Z, et al. Diagnostic performance of magnetic resonance elastography in staging liver fibrosis: a systematic review and meta-analysis of individual participant data. Clin Gastroenterol Hepatol 2015;13:440-451.e6.

16. Piscaglia F, Marinelli S, Bota S, et al. The role of ultrasound elastographic techniques in chronic liver disease: current status and future perspectives. Eur J Radiol 2014;83:450-455.

17. Deffieux T, Gennisson JL, Bousquet L, et al. Investigating liver stiffness and viscosity for fibrosis, steatosis and activity staging using shear wave elastography. J Hepatol 2015;62:317-324.

18. Leung VY, Shen J, Wong VW, et al. Quantitative elastography of liver fibrosis and spleen stiffness in chronic hepatitis B carriers: comparison of shear-wave elastography and transient elastography with liver biopsy correlation. Radiology 2013;269:910-918.

19. Korean Association for the Study of the Liver. KASL clinical practice guidelines: management of chronic hepatitis B. Clin Mol Hepatol 2016;22:18-75.

20. Bruix J, Sherman M; American Association for the Study of Liver Diseases. Management of hepatocellular carcinoma: an update. Hepatology 2011;53:1020-1022.

21. Xu X, Su Y, Song R, et al. Performance of transient elastography assessing fibrosis of single hepatitis B virus infection: a systematic review and meta-analysis of a diagnostic test. Hepatol Int 2015;9:558-566.

22. Wong GL, Chan HL, Wong CK, et al Liver stiffness-based optimization of hepatocellular carcinoma risk score in patients with chronic hepatitis B. J Hepatol 2014;60:339-345.

23. Jeong WK, Lim HK, Lee HK, Jo JM, Kim Y. Principles and clinical application of ultrasound elastography for diffuse liver disease. Ultrasonography 2014;33:149-160.

24. Lee HS, Kim JK, Cheong JY, et al. Prediction of compensated liver cirrhosis by ultrasonography and routine blood tests in patients with chronic viral hepatitis. Korean J Hepatol 2010;16:369-375.

25. Zeng J, Liu GJ, Huang ZP, et al. Diagnostic accuracy of twodimensional shear wave elastography for the non-invasive staging of hepatic fibrosis in chronic hepatitis B: a cohort study with 
internal validation. Eur Radiol 2014;24:2572-2581.

26. Chen CJ, Yang HI, Su J, et al. Risk of hepatocellular carcinoma across a biological gradient of serum hepatitis B virus DNA level. JAMA 2006;295:65-73.

27. Chang TT, Lai CL, Kew Yoon S, et al. Entecavir treatment for up to 5 years in patients with hepatitis B e antigen-positive chronic hepatitis B. Hepatology 2010;51:422-430.

28. Marcellin P, Gane E, Buti M, et al. Regression of cirrhosis during treatment with tenofovir disoproxil fumarate for chronic hepatitis
B: a 5-year open-label follow-up study. Lancet 2013;381:468-475. 29. Lee HW, Yoo EJ, Kim BK, et al. Prediction of development of liverrelated events by transient elastography in hepatitis B patients with complete virological response on antiviral therapy. Am J Gastroenterol 2014;109:1241-1249.

30. Lampertico P, Invernizzi F, Viganò M, et al. The long-term benefits of nucleos(t)ide analogs in compensated HBV cirrhotic patients with no or small esophageal varices: a 12-year prospective cohort study. J Hepatol 2015;63:1118-1125. 